$\underline{\text { Original Article }}$

\title{
PREPARATION OF CHITOSAN-TPP NANOPARTICLES: THE INFLUENCE OF CHITOSAN POLYMERIC PROPERTIES AND FORMULATION VARIABLES
}

\author{
N. K. AL-NEMRAWI*1 ${ }^{*}$ S. S. M. ALSHARIF², R. H. DAVE ${ }^{3}$
}

1Department of Pharmaceutical Technology $\backslash$ Faculty of Pharmacy $\backslash$ Jordan University of Science and Technology, Irbid 22110-Jordan, ${ }^{2}$ Department of Applied Biological Sciences $\backslash$ Faculty of Science and Art $\backslash$ Jordan University of Science and Technology, Irbid 22110-Jordan, ${ }^{3}$ Arnold and Marie Schwartz College of Pharmacy and Health Sciences, Pharmaceutical Sciences, 75 Dekalb Avenue Brooklyn, NY, USA 11201 Email: nknemrawi@just.edu.jo

Received: 31 Mar 2018, Revised and Accepted: 23 Jun 2018

\section{ABSTRACT}

Objective: The aim of this work was to prepare chitosan nanoparticles (CS NPs) using sodium tripolyphosphate (TPP) as crosslinker and to study the effect of chitosan polymeric properties and experimental conditions on the properties and stability of NPs.

Methods: CS NPs were prepared by ionic gelation method, using TPP as a crosslinker. The particle size (PS), polydispersity index (PDI), zeta potential (ZP) and the morphologies of the NPs were studied. CS NPs prepared by varying the concentration of TPP, Chitosan molecular weight and its degree of deacetylation, the stirring speed, the rate of TPP addition and the freeze-drying method to study the effect of these variables on the NPs. The stability of the CS NPs was evaluated by storing aqueous suspensions of NPs and comparing the PS, PDI and ZP at the beginning and the end of the experiment.

Results: This study shows that the PS, ZP and dispersity of the NPs depend on the chitosan polymeric properties and experimental conditions. The NPs sizes range between 145.73 and $724.23 \mathrm{~nm}$. They all carried positive charges ranging between +4.32 and $+43.67 \mathrm{mV}$. Most of the NPs have the same sizes after freeze-drying, but showed higher monodispersity and ZP, indicating higher stability. After twenty days of studying the stability, the NPs that had low ZP showed a large increment in size in comparison to the highly charged NPs.

Conclusion: In conclusion, the polymeric properties and formulation variables in the ionic gelation method have a great influence on the CS NPs formed.

Keywords: Chitosan, TPP, Stability, Nanoparticles

(C) 2018 The Authors. Published by Innovare Academic Sciences Pvt Ltd. This is an open access article under the CC BY license (http://creativecommons.org/licenses/by/4.0/) DOI: http://dx.doi.org/10.22159/ijap.2018v10i5.26375

\section{INTRODUCTION}

Chitosan (CS) is a polysaccharide that is used widely in pharmaceutical and biomedical preparations due to its unique biological properties. It is a safe, biocompatible, and biodegradable polymer. It has been approved by the food and drug administration (FDA) for wound dressing [1].

Chitosan is a linear copolymer of $\beta(1 \rightarrow 4)$ with randomly distributed $\mathrm{N}$-acetyl glucosamine and glucosamine units. Chitosan is a basic polysaccharide with a pKa around 6.5, therefore, it is only dissolved in an acidic condition. Such acidic conditions may affect the chitosan toxicity on cells, especially if it is going to be applied for long times $[2,3]$.

The amine groups in the chitosan structure get charged in acidic media making the polymer a positively charged polyelectrolyte. The positive charges carried by chitosan interact strongly with many minerals and cells in the body that carry negative charges such as cholesterol, fats, proteins, mucin and tumor cells. Furthermore, chitosan can makes electrostatic complexes that are used for drugs encapsulation $[4,5]$.

Chitosan has been used to prepare nanoparticles (NPs) by itself or to enhance other polymeric NPs properties. CS NPs loaded with different drugs were prepared and they proved to enhance the absorption of these agents from different routes such as nasal, gastrointestinal and transdermal routes [6-8]. Furthermore, chitosan was used to coat NPs prepared from other polymers to enhance the properties of these NPs. Chitosan is reported to enhance the permeability of other polymeric NPs, decrease phagocytosis in blood, increase NPs residence time in the site of application, harmonize their release kinetics or charge, and enhance their mucoadhesive properties [9-13].

Chitosan nanoparticles (CS NPs) have been prepared by different methods such as emulsion cross linking, spray drying, reverse micellar method, template polymerization, polyelectrolyte complex, precipitation and ionotropic gelation methods $[1,4]$.
Ionic gelation technique is basically based on the ionic interaction between the positively charged amino groups of chitosan and the negatively charged groups of a polyanion. This polyanion is usually called a crosslinker. Different crosslinkers have been used, among which is sodium tripolyphosphate (TPP) [14-16]. TPP is the most extensively used crosslinker in preparing CS NPs because of its safety and multivalent properties. Polyphosphates including TPP are hydrolyzed into simpler phosphates, which are considered as nutritious. Because of that, the toxicity of polyphosphates is very low, and they are considered safe. In addition, TPP have been reported to be neither mutagenic nor carcinogenic $[17,18]$.

In nanomedicine, the particle properties such as the size and the charge play an important role in determining its biological behavior. These properties affect the cellular uptake, protein adsorption, and accumulation of the NPs and their distribution throughout the body. Unfortunately, chitosan/TPP nanoparticles are usually polydispersed and suffer from poor stability, which limit their use. The particle size distribution depends on several factors such as the chitosan/TPP mixing, chitosan concentration, degree of deacetylation and molecular weight, the ionic strength and the media $\mathrm{pH}[19,20]$. These problems must be overcome before CS NPs can move to the clinical trials level and then become commercially available.

In this study, the effect of some factors on the size, polydispersity and charge of CS NPs in the presence of TPP as the crosslinkers were studied. The effect of the concentration of TPP, Chitosan molecular weight and its degree of deacetylation, the stirring (speed and method), the rate of TPP addition and the freeze-drying method on NPs were investigated. Finally, the prepared CS NPs were evaluated in vitro to determine their stability.

\section{MATERIALS AND METHODS}

\section{Materials}

Chitosan (250 kDa and 93.0\% DDA) was purchased from ShanghiHanshare Industry (Shanghai, China). Low molecular weight chitosan (50-190 kDa, 75\% DDA) was purchased from 
Sigma-Aldrich (St. Louis, MO). Sodium tripolyphosphate (TPP) was purchased from AZchm (china). All other chemicals and reagents used were of analytical grade.

\section{Methods}

\section{Preparation of different molecular weights of chitosan}

Two molecular weights of chitosan were used in this work, the first one was purchased from sigma and has a molecular weight of 50 $\mathrm{kDa}$. The other one was prepared in our laboratory by acid depolymerization and has a molecular weight of $15 \mathrm{kDa}$ [21]. This polymer was a result of cleaving high molecular weight chitosan of $250 \mathrm{kDa}$. Hight molecular chitosan was dissolved in $2 \mathrm{M} \mathrm{HCl}$ to make $1 \%$ solution. Then the solution was refluxed for two hours. After that, the solution was added to ethanol in a ratio of $1: 2 \mathrm{v} / \mathrm{v}$ and cooled down. Finally, the precipitate was filtered and washed several times with ethanol and centrifuged at $4000 \mathrm{rpm}$ for $5 \mathrm{~min}$ (Thermo scientific, Germany). The obtained chitosan was freeze dried for $24 \mathrm{~h}$ (Telstar, Spain) and the powder was kept in an amber-airtight glass bottle and stored at room temperature.

\section{Preparation of different DDA of chitosan}

Three different degrees of deacetylation (DDA) were used to prepare CS NPs in order to study the effect of chitosan DDA on the physicochemical properties of the NPs. All three patches have the same molecular weight, which is $15 \mathrm{kDa}$. The first one resulted from the previous step and has a DDA $\sim 92 \%$. This polymer was further used to prepare the other two DDAs by acetylation reaction. First, $1 \%$ CS (15 kDa) was dissolved in water at $\mathrm{pH}$ of 6.5 . Then the solution was divided into two parts to which acetic anhydride was added to make 1:0.15 and 1:0.6 molar ratios to prepare the 55\% and 75\% DDA, respectively. The mixtures were stirred at $500 \mathrm{rpm}$ at room temperature and the $\mathrm{pH}$ of the solutions was maintained at 6.25. After $10 \mathrm{~min}$, the chitosan solution was dialyzed against $4 \mathrm{~L}$ of distilled water with gentle stirring at room temperature for $24 \mathrm{~h}$ using dialysis tubes $(12,000-14,000$ Da molecular weight cut-off). Finally, the dialyzed chitosan solution was poured in Petri dishes and dried overnight in an oven at $40{ }^{\circ} \mathrm{C}$ and the powder was transferred to an amber-airtight glass bottle and stored at room temperature [21].

\section{Characterization of chitosan molecular weight}

The average molecular weight of chitosan was calculated by applying the Mark-Houwink's equation [22]. Briefly, chitosan was dissolved in water and the viscosity was measured using a viscometer (Vibro viscometer, Japan). Then, the viscosity average molecular weight was calculated.

\section{Characterization of chitosan DDA}

The DDA of the chitosan patches was determined using proton nuclear magnetic resonance ( ${ }^{1} \mathrm{H}-\mathrm{NMR}$ ) (Bruker Avance Ultra Shield $300 \mathrm{MHz}, \mathrm{USA})$. Chitosan samples ( $30 \mathrm{mg} / \mathrm{ml}$ in $\mathrm{D}_{2} \mathrm{O}$ ) were scanned 25 times. The DDA was calculated using the integrals of the $\mathrm{H}^{1}$ proton peak of the deacetylated monomer $\left(\mathrm{H}^{1}-\mathrm{D}\right)$ at $5.2 \mathrm{ppm}$ and the peak of the three protons of the acetyl group (H-Ac) at $2.8 \mathrm{ppm}$ [23].

\section{Preparation of CS NPs}

The NPs were prepared using a modified ionic gelation method previously described in the literature with some modifications. Chitosan was dissolved in an aqueous solution of acetic acid $(0.2$ $\mathrm{mg} / \mathrm{ml}$ ) to form $0.5 \mathrm{mg} / \mathrm{ml}$ of chitosan solution. The chitosan solution was stirred overnight at room temperature using a magnetic stirrer at a $\mathrm{pH}$ of 3.6. After that, the chitosan solution passed through a syringe filter $(0.45 \mu \mathrm{m})$, and was then preheated in a water bath at 60 for $10 \mathrm{~min}$. In another beaker, TPP was dissolved in HPLC water in different concentrations and then passed through a syringe filter $(0.45 \mu \mathrm{m})$ and cooled to $2-4$ l. Finally, $5 \mathrm{ml}$ of TPP solution was added to $10 \mathrm{ml}$ of chitosan solution and stirred for $10 \mathrm{~min}$. The formed suspension was centrifuged (Thermo scientific, Germany) for $30 \mathrm{~min}$ at $10000 \mathrm{rpm}$. The nanoparticles were repeatedly washed with deionized water and freeze-dried for $48 \mathrm{~h}$ at $-80^{\circ} \mathrm{C}$ to obtain the powdered nanoparticles [24,25].

This procedure was repeatedly used to prepare CS nanoparticles, and in each time, one of the preparation factors was changed in order to investigate its effect on the physicochemical properties of the NPs. TPP concentration, stirring speed, molecular weight of CS, CS degree of deacetylation and rate of adding TPP were investigated.

\section{Characterization of CS NPs}

The mean particle size (PS), polydispersity index (PDI) and zeta potential (ZP) of the nanoparticles were measured using a Zetasizernano ZS90 instrument (Malvern, UK). The particle sizes and PDI were determined at $25^{\natural} \mathrm{C}$ using dynamic light scattering (DLS). The zeta potential was calculated from the electrophoretic mobility of the NPs in aqueous solution using the Helmholtz-Smoluchowski equation under an electrical field of $40 \mathrm{~V} / \mathrm{cm}$. All measurements were carried out in triplicate $(n=3)$.

The morphologies of all CS nanoparticles were investigated using SEM (Thermo scientific, Germany). The samples were coated with carbon film prior to analysis, and then, studied under a microscope.

The stability of the CS nanoparticles was evaluated by storing aqueous suspensions of different formulas at room temperature $\left(\sim 25^{\circ} \mathrm{C}\right)$. Few milligrams of each NP formulation were dispersed in deionized water in a transparent glass bottle and observed for twenty days. The particle size, PDI, and the zeta potential were measured at the beginning and the end of the experiment.

\section{RESULTS AND DISCUSSION}

\section{Preparation of different DDA and molecular weights of chitosan}

The molecular weight and DDA of the chitosan purchased from sigma were determined and found to be $51.7 \pm 3.20 \mathrm{kDa}$ and $75.01 \pm 1.34 \%$, respectively. This polymer molecular weight will be referred to as $50 \mathrm{kDa}$ and it's DDA as $75 \%$ for simplicity. The chitosan that was prepared using high molecular weight chitosan had a molecular weight of $15.32 \pm 0.24 \mathrm{kDa}$ and a DDA of $91.9 \pm 0.22$ $\%$. Further, the other two patches prepared using low molecular weight chitosan had DDA values of $75.09 \pm 1.37 \%$ and $54.34 \pm 2.16 \%$. The molecular weight of these polymers will be referred to as 15 $\mathrm{kDa}$ and their DDAs as $90 \%, 75 \%$ and $50 \%$ for simplicity.

\section{Preparation of CS NPs}

Different patches of CS NPs were prepared by changing a single factor at each time. The effect of TPP concentration, stirring speed, the molecular weight of CS, CS DDA and rate of TPP addition were investigated and the mean particle size, PDI and zeta potential of the different formulations were measured (table 1). All NPs prepared in this work showed spherical morphology confirmed by scanning electron microscope (SEM) (fig. 1). Following is the effect of each factor on nanoparticles physicochemical properties.

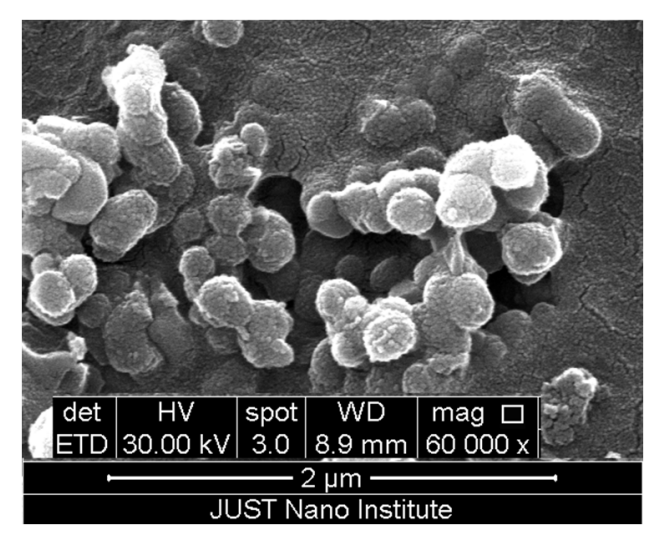

Fig. 1: SEM image of CS NPs with a scale bar of $2 \mu \mathrm{m}$

\section{Effect of TPP Concentration on CS NPs}

Different concentrations of TPP were used and their effects on the size, PDI and charge of the nanoparticles were investigated. As the 
amount of TPP used in the formulation increases, the size of the NPs decreases to a minimum and then starts to increase again (fig. 2-A). In this study the amount of the TPP was increased while the amount of chitosan and the volumes of the liquids were constant. In general, crosslinking agents are used in CS NPs formulation to reduce the mobility of chitosan and enhance its physicochemical properties such as stability. TPP is a polyanion that interacts with the amino groups in chitosan to form a gel through inter-and intra-molecular cross-linkages during the ionic gelation procedure [16].

Table 1: CS NPs, prepared under different conditions, mean particles size (nm), PDI and ZP (mV) before and after freeze-drying (n=3)

\begin{tabular}{|c|c|c|c|c|c|c|c|c|c|c|c|}
\hline \multirow[b]{2}{*}{ Formula } & \multirow[b]{2}{*}{ TPP (mg) } & \multirow[b]{2}{*}{ CS MWT $^{*}$} & \multirow[b]{2}{*}{ CS DDA } & \multirow[b]{2}{*}{ TPP addition ${ }^{\#}$} & \multirow[b]{2}{*}{ Stirring $^{+}$} & \multicolumn{3}{|c|}{ Before freeze drying } & \multicolumn{3}{|c|}{ After freeze drying } \\
\hline & & & & & & PS (nm) & PDI & $\mathrm{ZP}(\mathrm{mV})$ & PS (nm) & PDI & $\mathrm{ZP}(\mathrm{mV})$ \\
\hline F1 & 7.5 & 50 & 90 & 0.25 & 700 & 208.50 & 0.08 & 17.37 & 169.00 & 0.20 & 20.33 \\
\hline F2 & 7.5 & 50 & 90 & 0.50 & 700 & 277.20 & 0.10 & 15.67 & 186.90 & 0.22 & 8.04 \\
\hline F3 & 7.5 & 50 & 90 & 1.00 & 700 & 247.13 & 0.15 & 18.13 & 229.80 & 0.27 & 6.06 \\
\hline F4 & 7.5 & 50 & 90 & 1.50 & 700 & 257.93 & 0.22 & 6.53 & 254.63 & 0.24 & 12.80 \\
\hline F5 & 7.5 & 15 & 90 & 2.50 & 700 & 388.07 & 0.41 & 8.14 & 346.13 & 0.50 & 13.73 \\
\hline F7 & 7.5 & 15 & 80 & 2.50 & 700 & 555.10 & 0.35 & 7.00 & 446.13 & 0.41 & 10.63 \\
\hline F6 & 7.5 & 15 & 55 & 2.50 & 700 & 663.07 & 0.51 & 4.78 & 582.07 & 0.52 & 4.32 \\
\hline F8 & 7.5 & 50 & 90 & 2.50 & 500 & 383.97 & 0.47 & 27.13 & 350.47 & 0.48 & 30.31 \\
\hline F9 & 7.5 & 50 & 90 & 2.50 & 1000 & 287.40 & 0.22 & 5.69 & 262.23 & 0.25 & 10.90 \\
\hline F10 & 7.5 & 50 & 90 & 2.50 & 1500 & 145.73 & 0.28 & 8.74 & 243.37 & 0.22 & 12.23 \\
\hline F11 & 1.5 & 50 & 90 & 2.50 & 700 & 610.00 & 0.54 & 40.00 & 576.13 & 0.54 & 43.67 \\
\hline F12 & 2.0 & 50 & 90 & 2.50 & 700 & 400.00 & 0.55 & 39.00 & 444.60 & 0.55 & 43.53 \\
\hline F13 & 3.0 & 50 & 90 & 2.50 & 700 & 356.70 & 0.28 & 30.50 & 295.87 & 0.12 & 35.30 \\
\hline F14 & 7.5 & 50 & 90 & 2.50 & 700 & 296.83 & 0.30 & 24.83 & 358.50 & 0.22 & 33.00 \\
\hline F15 & 15.0 & 50 & 90 & 2.50 & 700 & 508.43 & 0.28 & 5.38 & 540.80 & 0.21 & 8.16 \\
\hline F16 & 30.0 & 50 & 90 & 2.50 & 700 & 329.60 & 0.25 & 35.90 & 724.23 & 0.37 & 37.00 \\
\hline
\end{tabular}

* CS molecular weight in $\mathrm{kDa}$. \#TPP addition rate in $\mathrm{ml} / \mathrm{min}$. + Stirring the rate in $\mathrm{rpm} . \mathrm{n}=3$ and data are given in mean \pm SD.

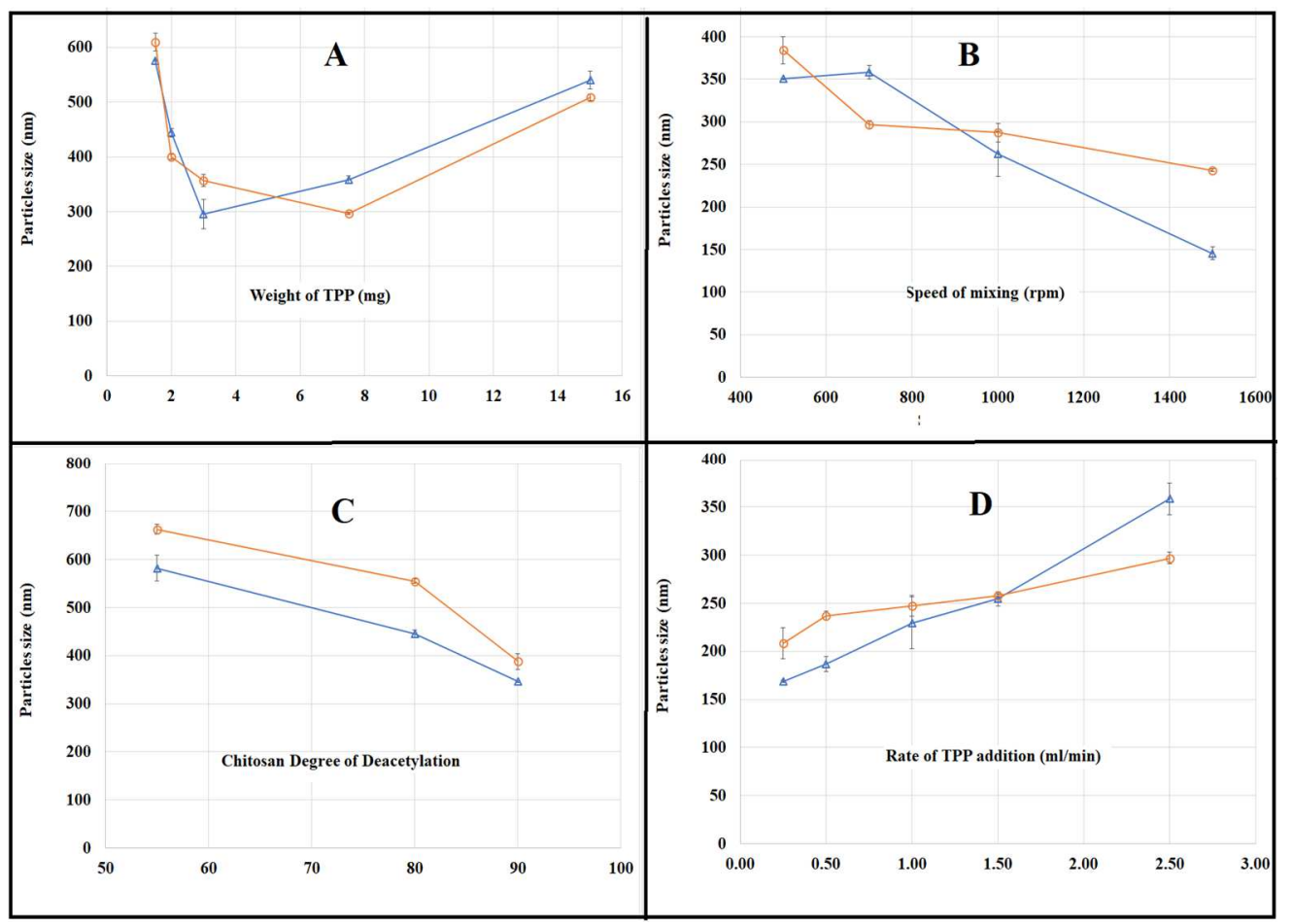

Fig. 2: The effect of different variables on CS NPs size (nm) before and after freeze-drying:(A) Effect of TPP amount (mg), (B) Effect of speed of mixing (rpm), (C) Effect of chitosan DDA and (D) Effect of TPP addition rate(ml/min). $\mathrm{n}=3$ and data are given in $\mathrm{mean} \pm \mathrm{SD}$.

As the amount of TPP increases, the nanoparticle suspension became progressively more turbid due to the formation of chitosan nanoparticles. When TPP amount was very low, the reaction solution was clear and viscous like pure chitosan solution, which indicated that the TPP amount was not enough to form a cross-linked structure of chitosan. As TPP amount increased to more than $1.5 \mathrm{mg}$, the NPs started to be formed, and as the amount of TPP increased, the particle sizes decreased.
This may be due to increased inter and intra cross-linking between chitosan and TPP. On the other hand, as the amount of TPP continued to increase to more than $3 \mathrm{mg}$, CS NPs size started to increase. This may be due to the fact that after chitosan molecules were fully crosslinked, the excess TPP would lead to more chitosan molecules being involved in the formation of a single nanoparticle [4, 26]. In addition, as TPP concentration increases the samples become more monodispersed, and this is indicated by PDI values (fig. 3-A). 


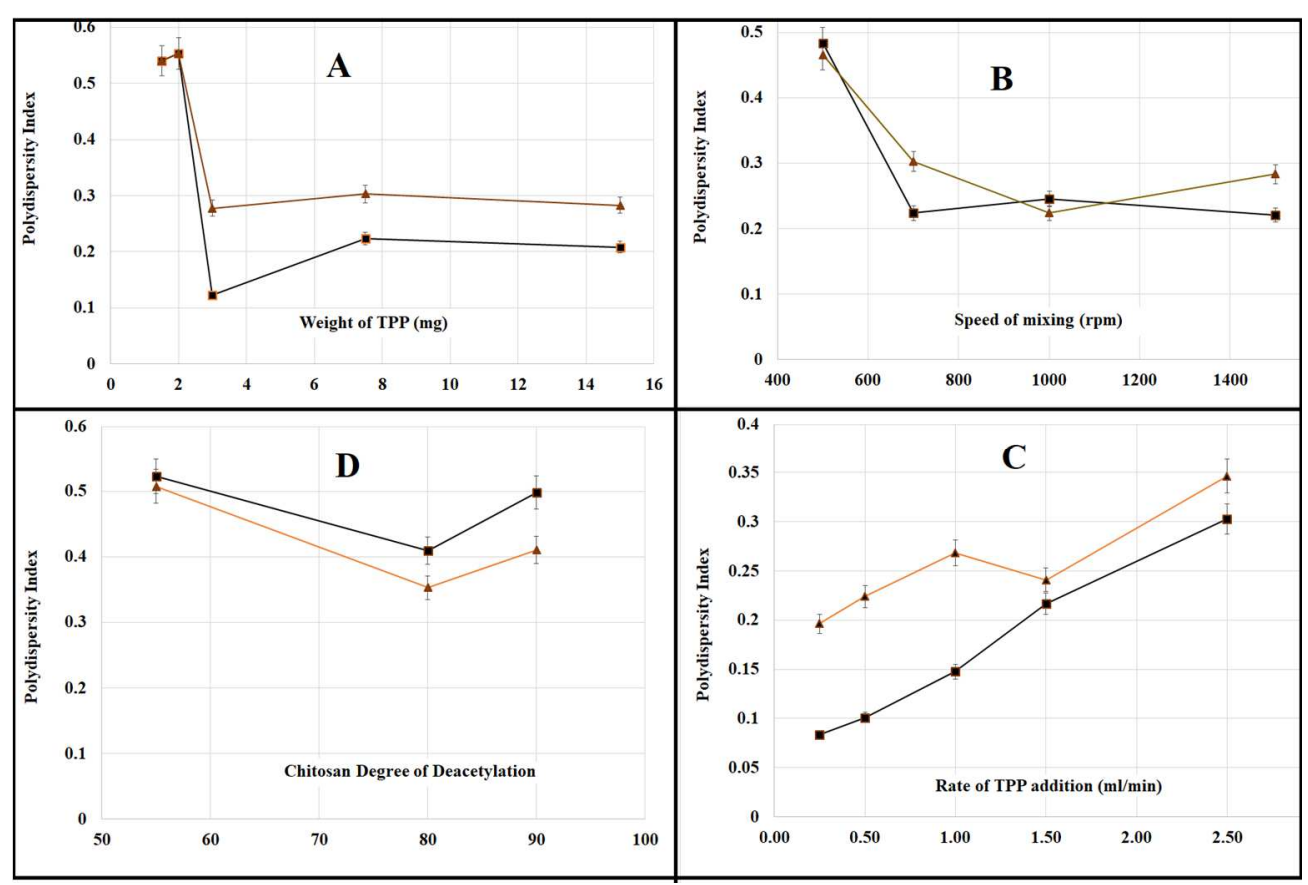

Fig. 3: The effect of different variables on CS NPs PDI before and after freeze-drying:(A) Effect of TPP amount (mg), (B) Effect of speed of mixing (rpm), (C) Effect of chitosan DDA and (D) Effect of TPP addition rate(ml/min). $\mathrm{n}=3$ and data are given in mean \pm SD.

In general, nanosuspensions having high positive charges above +10 or below-10 are expected to have good stability while suspensions with charges between-10 and+10 are expected to have a higher possibility of aggregation and less stability. All nanoparticles prepared in this work carried positive charges.
The charges of the nanoparticles decrease as the TPP concentration increases, which can be related to the neutralization of the protonated amino groups on chitosan by TPP anions (fig. 4-A). All formulas prepared with different TPP amounts, except F15, had surface charges above +10 , which indicates the high stability [27].

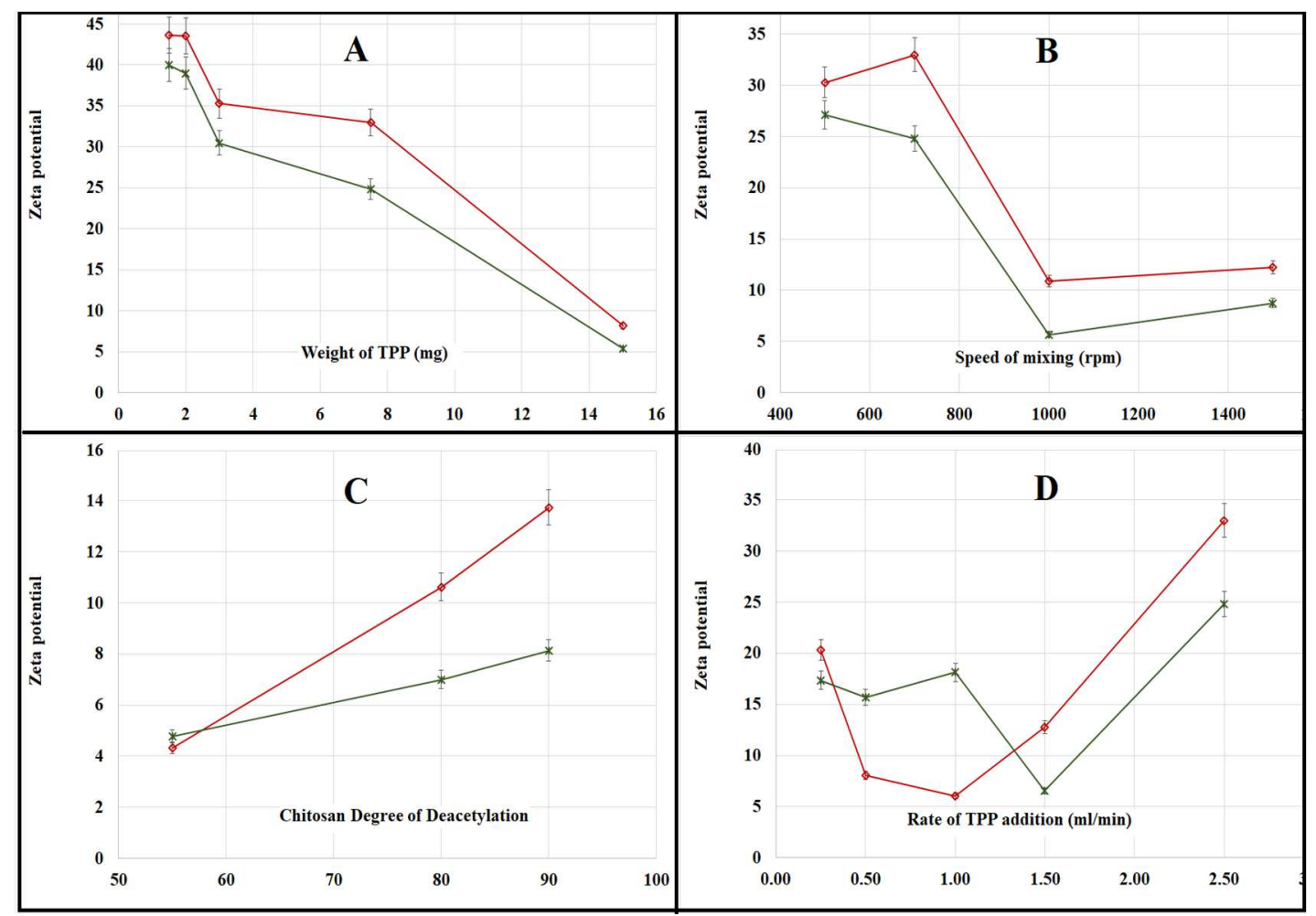

Fig. 4: The effect of different variables on CS NPs zeta potential $(\mathrm{mV})$ beforeand after freeze-drying:(A) Effect of TPP amount (mg), (B) Effect of speed of mixing (rpm), (C) Effect of chitosan DDA and (D) Effect of TPP addition rate (ml/min). $\mathrm{n}=3$ and data are given in mean \pm SD. 


\section{Effect of the speed of stirring on CS NPs}

The speed of stirring effect on particle size was also investigated and the results showed that the particle size decreased as the speed increased from $500 \mathrm{rpm}$ to $1500 \mathrm{rpm}$ (fig. 2-B). This trend is probably due to the increase of the shear force that accelerates the dispersion of TPP in chitosan solution. The monodispersity of the nanoparticles was improved as the stirring speed increased, which is expected, as a consequence of the better dispersion of TPP in chitosan solution (fig. 3-B). The surface charge of the nanoparticles was also affected by the stirring speed, where the higher the speed was, the lower was the charge (fig. 4-B). This observation may be related to the better dispersion of TPP in chitosan solution that led to more inter and intra cross-linkages in chitosan at higher speeds.

\section{Effect of CS molecular weight on CS NPs}

Two different chitosan molecular weights ( 15 and $50 \mathrm{kDa}$ ) were tested to investigate the effect of the molecular weight of chitosan on CS NPs properties. Under the same conditions, the nanoparticles sizes increased as the molecular weight of chitosan decreases. This effect of chitosan's molecular weight may be related to the viscosity of the external phase. As the chitosan molecular weight increases, the viscosity of the solution increases, which leads to smaller NPs. On the other hand, both the PDI and zeta potential decreased as the molecular weight increased. This can be explained by referring to the length of the strand and its relation to the positive charge density. As the length of the strand decreases, the number of amine groups per strand also decreases. Since the amine groups are responsible for the positive charge, a sharp decrease of the zeta potential is expected as the molecular weight decreases. In general, the PDI in all NPs prepared using the low molecular weight chitosan was higher than those prepared with high molecular weight chitosan.

\section{Effect of CS DDA on CS NPs}

Three degrees of deacetylation of chitosan $(55,80,90 \%)$ were investigated and the effect of DDA on PS, PDI and ZP was investigated (fig. 2-C, fig. 3-Cand fig.4-C). The results showed that as the DDA increased the particle size decreased. The DDA value is related to the percentage of the deacetylated amine groups along the molecular chain, and it is increased as the percentage of amine groups to acetylated groups increased. Therefore, the DDA value of chitosan determines the density of the positive charge that is expressed as the zeta potential [3]. The large positive charge density increases the potential of the chitosan molecules to cross-link with TPP. This eventually led to smaller NPs, as we mentioned previously. The PDI did not show a clear relation to chitosan DDA, but in general the samples prepared were less monodispersed, which may be related to the CS molecular weight since all NPs prepared using CS $15 \mathrm{kDa}$ have higher PDIs in comparison to NPs prepared using CS 50 $\mathrm{kDa}$. Increasing the DDA value of chitosan results in a higher density of the positive charge, which is expected to increase the zeta potential. Our results showed such behavior.

\section{Effect of TPP addition rate on CS NPs}

Moreover, the effect of TPP addition rate on nanoparticles properties was investigated. The flow rate of the TPP solution was between 0.25 and $2.5 \mathrm{ml} / \mathrm{min}$. As the TPP solution flow rate increases, the particle size increases, as well as the PDI (fig.2-D and fig.3-D). This could be explained by the higher concentration of TPP at the interface between the two solutions at higher rates of addition. Consequently, this led to higher inter and intra cross-linkages that consequently produced larger particles. The zeta potential results showed a decrease at the beginning; then it started to increase (fig. 4-D).

\section{Effect of freeze-drying on CS NPs}

It is well known that upon liquid removal from nanoparticles, they would agglomerate and grow up so that the huge specific surface energy is overcome. Freeze-drying is a process that is widely used for drying NPs to improve their stability. It is considered as a relatively slow and expensive method. In freeze-drying, the materials are freezed and then the surrounding pressure is reduced to allow the frozen liquids in the material to sublime directly from the solid phase to the gas phase[28,29]. This process is typically used to preserve delicate materials such as NPs. The effect of freeze-drying on the CS NPs size, PDI and ZP were studied and the results are summarized in table 1 and in fig. 2,3 and 4. Freeze-drying is expected to decrease the NPs size because it prevents the nanoparticles from agglomeration. Some of the NPs prepared in this work showed such behavior but the majority of the NPs have the same sizes before and after freeze-drying. The short time difference between measuring the NPs size before and after freezing may be the reason behind this observation. In general, the PDIs were lower after freeze-drying and the surface charges were higher. This may indicate that freeze-drying enhanced the NPs stability.

\section{Stability study}

The freeze-dried NPs were dispersed in deionized water and observed over twenty days in order to evaluate their stability. In general, all NPs suspensions did not change in appearance or get turbid. On the other hand, in certain cases, there were differences in the NPs sizes at the beginning of the study and after the twenty days elapsed. There is a clear relation between the surface charge and the particles size increment percentage (fig. 5). The particles size increment percentage was calculated as follows:

Size increment

$$
=\frac{\text { Size at the begining of the study }- \text { size after } 20 \text { days }}{\text { Size at the begining of the study }} \text { X100\% }
$$

When the NPs carry high positive charges, the suspensions are more stable, and the NPs sizes remain almost the same. On the contrast, the lower the surface charges are, the lower is the stability that is indicated by the size growth.

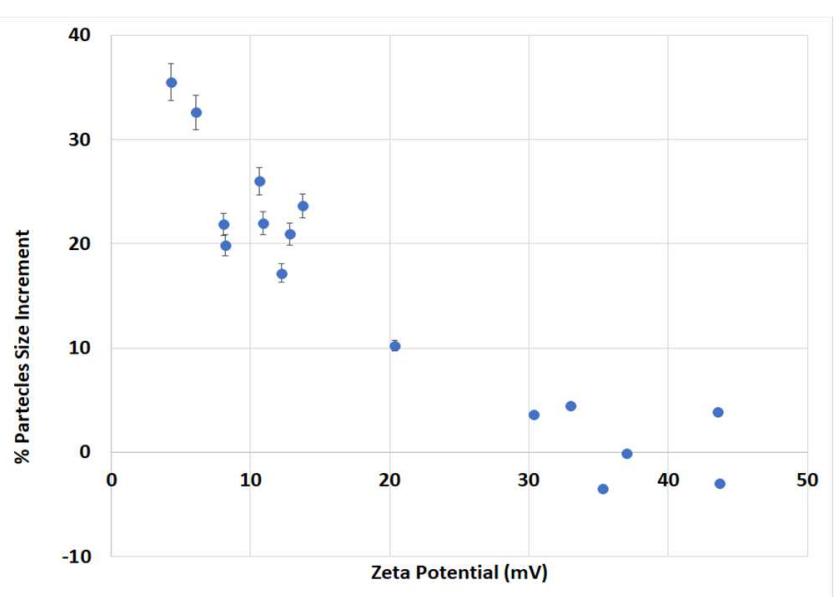

Fig. 5: The relationship between particles $\mathrm{ZP}(\mathrm{mV})$ and the particles size increment percentage. $\mathrm{n}=3$ and data are given in mean \pm SD 


\section{CONCLUSION}

CS NPs properties such as size, polydispersity and charge affect their biological properties and stability. The NPs size decreases then increases as the amount of TPP increases but it decreases as both the stirring rate or chitosan molecular weight increases. Also, NPs size increases as TPP flow rate decreases. The NPs are more monodispersed at higher TPP concentrations, stirring rates, chitosan molecular weights or TPP flow rates, but they show no relation to chitosan DDA. All NPs have positive charges that decrease at higher TPP concentrations, stirring rates or chitosan molecular weights or lower chitosan DDA. ZP decreased, then increased as TPP flow rate increased. Most of the NPs have the same sizes but have a higher monodispersity and ZP after freeze-drying. The NPs stability shows a clear relation to the NPs surface charge and it increases as the charge becomes more positive. In conclusion, the polymeric properties and formulation variables in ionic gelation method has a great influence on the CS NPs formed.

\section{ACKNOWLEDGMENT}

The authors thank the deanship of research at Jordan University of Science and Technology (JUST) for their support and using the facilities and instruments in the school.

\section{AUTHORS CONTRIBUTIONS}

All the author have contributed equally

\section{CONFLICTS OF INTERESTS}

All authors have none to declare

\section{REFERENCES}

1. Agnihotri SA, Mallikarjuna NN, Aminabhavi TM. Recent advances on chitosan-based micro-and nanoparticles in drug delivery. J Controlled Release 2004;100:5-28.

2. Harish Prashanth KV, Tharanathan RN. Chitin/chitosan: modifications and their unlimited application potential-an overview. Trends Food Sci Technol 2007;18:117-31.

3. Pillai CKS, Paul W, Sharma CP. Chitin and chitosan polymers: chemistry, solubility and fiber formation. Prog Polym Sci 2009;34:641-78.

4. Fan W, Yan W, Xu Z. Formation mechanism of monodisperse, low molecular weight chitosan nanoparticles by ionic gelation technique. Colloids Surf B 2012;90:21-7.

5. Ilium L. Chitosan and Its use as a pharmaceutical excipient. Pharm Res 1998;15:1326-31.

6. Campos AM de, Diebold Y, Carvalho ELS. Chitosan nanoparticles as new ocular drug delivery systems: in vitro stability, in vivo fate, and cellular toxicity. Pharm Res 2004:21:803-10.

7. Dyer AM, Hinchcliffe M, Watts P. Nasal delivery of insulin using novel chitosan-based formulations: a comparative study in two animal models between simple chitosan formulations and chitosan nanoparticles. Pharm Res 2002;19:998-1008.

8. Sun Y, Wan A. Preparation of nanoparticles composed of chitosan and its derivatives as delivery systems for macromolecules. J Appl Polym Sci 2007;105:552-61.

9. Cui $\mathrm{F}, \mathrm{He} \mathrm{C}, \mathrm{He} \mathrm{M}$. Preparation and evaluation of chitosan-ethylenediaminetetraacetic acid hydrogel films for the mucoadhesive transbuccal delivery of insulin. J Biomed Mater Res A 2009;89:1063-71.
10. Chakravarthi SS, Robinson DH. Enhanced cellular association of paclitaxel delivered in chitosan-PLGA particles. Int J Pharm 2011;409:111-20.

11. Thanou M, Florea BI, Geldof M. Quaternized chitosan oligomers as novel gene delivery vectors in epithelial cell lines. Biomaterials 2002;23:153-9.

12. Takeuchi I, Takeshita T, Suzuki T. Iontophoretic transdermal delivery using chitosan-coated PLGA nanoparticles for positively charged drugs. Colloids Surf B 2017;160:520-6.

13. Varshosaz J, Taymouri S, Hamishehkar H. Fabrication of polymeric nanoparticles of poly(ethylene-co-vinyl acetate) coated with chitosan for pulmonary delivery of carvedilol. J Appl Polym Sci 2014;131. https://doi.org/10.1002/app.39694

14. Wu J, Wang Y, Yang H. Preparation and biological activity studies of resveratrol loaded ionically cross-linked chitosan-TPP nanoparticles. Carbohydr Polym 2017;175:170-7.

15. Jing ZW, Jia YY, Wan N. Design and evaluation of novel pH-sensitive ureido-conjugated chitosan/TPP nanoparticles targeted to helicobacter pylori. Biomaterials 2016;84:276-85.

16. Lee E, Park SJ, Lee JH. P-049-preparation of chitosan-TPP nanoparticles and their physical and biological properties. Asian J Pharm Sci 2016;11:166-7.

17. Kulakovskaya TV, Vagabov VM, Kulaev IS. Inorganic polyphosphate in industry, agriculture and medicine: modern state and outlook. Process Biochem 2012;47:1-10.

18. Nagpal K, Singh SK, Mishra DN. Optimization of brain-targeted chitosan nanoparticles of rivastigmine for improved efficacy and safety. Int J Biol Macromol 2013;59:72-83.

19. Qinna NA, Karwi QG, Al-Jbour N. Influence of molecular weight and degree of deacetylation of low molecular weight chitosan on the bioactivity of oral insulin preparations. Mar Drugs 2015;13:1710-25.

20. Taetz S, Nafee N, Beisner J. The influence of chitosan content in cationic chitosan/PLGA nanoparticles on the delivery efficiency of antisense $2^{\prime}$-O-methyl-RNA directed against telomerase in lung cancer cells. Eur J Pharm Biopharm 2009;72:358-69.

21. Obaidat R, Al-Jbour N, Al-Sou'd K. Some physicochemical properties of low molecular weight chitosans and their relationship to conformation in aqueous solution. J Solut Chem 2010;39:575-88.

22. Elsayed A, Remawi MA, Qinna N. Formulation and characterization of an oily-based system for oral delivery of insulin. Eur J Pharm Biopharm 2009;73:269-79.

23. Qandil AM, Obaidat AA, Ali MAM. Investigation of the interactions in complexes of low molecular weight chitosan with ibuprofen. J Solut Chem 2009;38:695-712.

24. Manimekalai P, Dhanalakshmi R, Manavalan R. Preparation and characterization of ceftriaxone sodium encapsulated chitosan nanoparticles. Int J Appl Pharm 2017;9:10-5.

25. Ojha S, Kumar B. Formulation and optimization of chitosan nanoparticles of dimethyl fumarate using box-behnken design. Int J Appl Pharm 2016;8:10-7.

26. Thandapani G, PSP, PNS. Size optimization and in vitro biocompatibility studies of chitosan nanoparticles. Int J Biol Macromol 2017;104:1794-806.

27. Retamal Marin RR, Babick F, Hillemann L. Zeta potential measurements for non-spherical colloidal particles-practical issues of characterisation of interfacial properties of nanoparticles. Colloids Surf A 2017;532:516-21.

28. Choi MJ, Briançon S, Andrieu J. Effect of freeze-drying process conditions on the stability of nanoparticles. Dry Technol 2004;22:335-46.

29. Abdelwahed W, Degobert G, Stainmesse S. Freeze-drying of nanoparticles: formulation, process and storage considerations. Adv Drug Delivery Rev 2006;58:1688-713. 